\title{
Lesson of the month 1: A rare adverse reaction between flucloxacillin and paracetamol
}

\author{
Authors: William Osborne, ${ }^{A}$ Aneeka Chavda, ${ }^{B}$ George Katritsis ${ }^{C}$ and Jon S Friedland ${ }^{D}$
}

Flucloxacillin, a beta-lactam antibiotic, is a commonly prescribed antibiotic for the treatment of infections caused by staphylococci and streptococci, most notably Staphylococcus aureus. Paracetamol is one of the most dispensed medications by NHS England and is used for the treatment of fever and pain. ${ }^{1}$ However most doctors are unaware that concurrent use of these drugs can cause a potentially fatal drug interaction due to pyroglutamic acidosis (PGA), also known as 5-oxoprolinaemia. PGA is a rare cause of raised anion gap metabolic acidosis due to disruption of the $\gamma$-glutamyl cycle. We report the case of a patient with multiple comorbidities who developed PGA due to coadministration of paracetamol and flucloxacillin.

KEYWORDS: Pyroglutamic acidosis, paracetamol, flucloxacillin, adverse drug reaction, 5-oxoprolinaemia, metabolic acidosis

An 86-year-old man presented to our emergency department with a several day history of confusion and increased urinary frequency (day 1). His past medical history included type 2 diabetes mellitus and hypertension. He had recently been admitted with methicillin-sensitive Staphylococcus aureus bacteraemia for which he received 2 weeks of intravenous flucloxacillin. His medications included biphasic insulin, metformin, mirtazapine, ramipril, sitagliptin, thiamine, vitamin $\mathrm{B}_{6}$ and calcium carbonate I vitamin D supplements with no known drug allergies. He was febrile at $38.2^{\circ} \mathrm{C}$ with a pulse rate of $100 \mathrm{bpm}$ and normal blood pressure. Clinical examination revealed only a hyperactive delirium and distended bladder. Blood testing showed a normocytic anaemia $(110 \mathrm{~g} / \mathrm{L})$, leukocytosis $\left(22.9 \times 10^{3} / \mathrm{mm}^{3}\right)$ with neutrophilia $\left(21.2 \times 10^{3} / \mathrm{mm}^{3}\right)$, a raised C-reactive protein $(215 \mathrm{mg} / \mathrm{L})$, a raised serum glucose $(31.2 \mathrm{mmol} / \mathrm{L})$ with associated raised serum osmolality (298 mmol/L), hyponatraemia (121 mmol/L), and

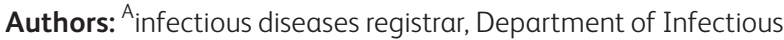
Diseases, Royal Stoke University Hospital, Stoke-on-Trent, UK; ${ }^{\mathrm{B}}$ lead pharmacist for infection, Imperial College Healthcare NHS Trust, London, UK; C Cardiology research fellow, Imperial College London, London, UK; D professor of infectious diseases and deputy principal, Institute of Infection and Immunity, St George's, University of London, London, UK acute renal failure (creatinine $199 \mathrm{mmol} / \mathrm{L}$ and urea $24.4 \mathrm{mmol} / \mathrm{L}$ ). Arterial blood gas analysis revealed partially compensated metabolic acidosis ( $\mathrm{pH} 7.34$, lactate $3.3 \mathrm{mmol} / \mathrm{L}$, base excess $-12.3 \mathrm{mmol} / \mathrm{L}$, bicarbonate $13.5 \mathrm{mmol} / \mathrm{L}$ and anion gap $21 \mathrm{mmol} / \mathrm{L})$.

The initial working diagnosis was Gram-negative sepsis complicated by acute kidney injury, hyperglycaemia, urinary retention and delirium. Intravenous crystalloid fluids, antibiotics (ceftriaxone $2 \mathrm{~g} /$ day and gentamicin $5 \mathrm{mg} / \mathrm{kg}$ once only), oral paracetamol ( $4 \mathrm{~g} /$ day), prophylactic low molecular weight heparin and a fixed-rate insulin infusion were started. A urinary Foley catheter was inserted. Blood cultures taken at presentation grew Staphylococcus aureus (methicillin-sensitive). Antibiotics were changed to intravenous flucloxacillin (12 g/day).

On day 29 , the patient became hypotensive and obtunded. Blood pressure was $60 / 40 \mathrm{mmHg}$, pulse rate $84 \mathrm{bpm}$, oxygen saturation $97 \%$ on room air and a respiratory rate $26 \mathrm{bpm}$. Clinical examination revealed bi-basal coarse crepitations and wheeze on auscultation of the chest. Arterial blood gas analysis showed a profound metabolic acidosis with raised anion gap $(\mathrm{pH} 6.99$, bicarbonate $3.9 \mathrm{mmol} / \mathrm{L}$, base excess $-27.5 \mathrm{mmol} / \mathrm{L}$, chloride $119 \mathrm{mmol} / \mathrm{L}$, calculated anion gap $28 \mathrm{mmol} / \mathrm{L}, \mathrm{PCO}_{2} 2.1 \mathrm{kPa}$, $\mathrm{PO}_{2} 14.5 \mathrm{kPa}$, lactate $1.1 \mathrm{mmol} / \mathrm{L}$ and glucose $10.6 \mathrm{mmol} / \mathrm{L}$ ). Urine ketones were negative. The patient was transferred to the intensive care unit. A provisional diagnosis of septic shock was made and antibiotics were changed to meropenem ( $1 \mathrm{~g}$ every 8 hours). Vasopressor support (using adrenaline, noradrenaline and vasopressin) was required to maintain adequate organ perfusion. An $8.4 \%$ sodium bicarbonate infusion was given whilst continuous veno-venous haemofiltration was initiated to correct the acidosis. The patient developed respiratory failure and required mechanical ventilation.

Diverse causes of a raised anion gap metabolic acidosis were excluded. The diagnosis of pyroglutamic acidosis (PGA) was considered and paracetamol discontinued. Following this one change in treatment, the patient rapidly improved, was extubated and weaned off vasopressors. After 7 days, flucloxacillin was restarted but paracetamol was not. The patient continued to improve without return of acidosis and was subsequently discharged home.

Acidaemia in this context results from the accumulation of 5-oxoproline, a natural amino acid derivative. The proposed mechanism is thought to be due to disorder of the $\gamma$-glutamyl cycle. ${ }^{2}$ Paracetamol creates a glutathione-deficient state through its metabolite, $\mathrm{N}$-acetyl benzoquinoneimine, which irreversibly 
Fig 1. The $\gamma$-glutamyl cycle, illustrating the proposed mechanism of 5-oxoprolinaemia as a result of paracetamol and flucloxacillin co-administration.

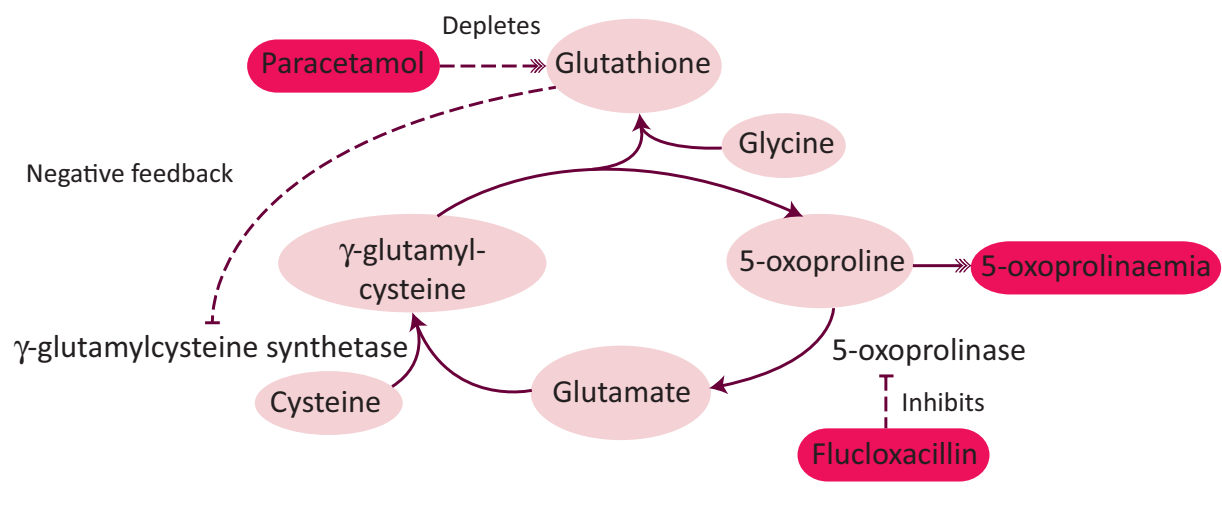

binds to glutathione. Low glutathione levels release the negative feedback inhibition on the enzyme $\gamma$-glutamylcysteine synthetase resulting in raised levels of $\gamma$-glutamylcysteine. This is converted to 5 -oxoproline, which is in turn degraded by 5-oxoprolinase (see Fig 1). Flucloxacillin inhibits 5-oxoprolinase leading to accumulation of 5-oxoproline, acidaemia and 5-oxoprolinuria. This disorder is encountered more frequently in neonates due to genetic defects in either glutathione synthetase or 5-oxoprolinase. This results in a spectrum of neonatal illness including haemolytic anaemia, seizures and other neurological disorders. ${ }^{3}$

Drug-induced PGA in adults is most commonly seen in chronic therapeutic paracetamol ingestion. This is in contrast to toxic paracetamol ingestion, which is well known to produce metabolic acidosis due to separate mechanisms (lactic acidosis or renal failure). This association was first reported in 1989 in a woman with neurological symptoms and haemolytic anaemia. ${ }^{4}$ Interestingly in this case the urine toxicology screen was positive for paracetamol however the link was only made between the two the following year. ${ }^{5}$ However other factors are often required to produce clinical or biochemically detectable PGA. These risk factors, by also promoting glutathione deficiency, include advanced age, female gender, malnourishment, renal failure, chronic liver failure, alcohol abuse and sepsis. ${ }^{6}$ The individual susceptibility of developing drug-induced PGA is most likely genetic, the prevalence of which in unknown. The majority of cases have shown a marked female preponderance. ${ }^{3,2}$ This could be explained in part by different in vitro activity of isoenzymes involved in the $\gamma$-glutamyl cycle between men and women. ${ }^{7}$ The interaction between flucloxacillin and paracetamol as a separate entity has been increasingly described in the literature. ${ }^{8}$ Other drugs such as vigabatran ${ }^{9}$ have also been implicated.

Diagnosis is often made by drug history, arterial acid-base analysis and exclusion of more common causes of raised anion gap acidosis. For definitive diagnosis, urine can be sent for detection of organic acids (which includes 5-oxoproline) by gas chromatography-mass spectrometry but this is not widely available. Treatment of PGA involves supportive care and stopping the offending drugs. The use of $\mathrm{N}$-acetylcysteine (NAC) has been proposed and used with success in some cases. ${ }^{10}$ NAC provides cysteine in the $\gamma$-glutamyl cycle, promoting glutathione synthesis. Whether prophylactic glutathione supplementation would prevent this still needs to be elucidated.
This case highlights the reaction between flucloxacillin and therapeutic-dose paracetamol resulting in PGA. With the increasing age and frailty of the general population and widespread use of paracetamol (20,152,891 prescriptions in 2017 in England), ${ }^{1}$ a large proportion of patients admitted to secondary care will be glutathione-deficient. Flucloxacillin is a commonly prescribed antibiotic creating multiple opportunities for this adverse reaction. Although this reaction is perceived to be relatively uncommon, it is almost certainly under-reported.

\section{References}

1 NHS Digital. Prescription cost analysis: England 2017. London: NHS, 2018. http://digital.nhs.uk/pubs/prescostanalysiseng2017 [Accessed 5 January 2019].

2 Dempsey GA, Lyall HJ, Corke CF, Scheinkestel CD. Pyroglutamic acidemia: A cause of high anion gap metabolic acidosis. Crit Care Med 2000;28:1803-7.

3 Emmett M. Acetaminophen toxicity and 5-oxoproline (pyroglutamic acid): A tale of two cycles, one an ATP-depleting futile cycle and the other a useful cycle. Clin J Am Soc Nephrol 2014;9:191-200.

4 Cress MH, Lau BWC, Jones ], Chan KM. Pyroglutamic acidemia in an adult patient. Clin Chem 1989;35:684-6.

5 Pitt J. Association between paracetamol and pyroglutamic aciduria. Clin Chem 1990;36:173-4.

6 Fenves AZ, Kirkpatrick HM 3rd, Patel VV et al. Increased anion gap metabolic acidosis as a result of 5-oxoproline (pyroglutamic acid): a role for acetaminophen. Clin J Am Soc Nephrol 2006;1:441-7.

7 Butera L, Feinfeld DA, Bhargava M. Sex differences in the subunits of glutathione-S-transferase isoenzyme from rat and human kidney. Enzyme 1990;43:176-82.

8 Myall K, Sidney J, Marsh A. Mind the gap! An unusual metabolic acidosis. Lancet 2011:377:526.

9 Bonham JR, Rattenbury JM, Meeks A, Pollit RJ. Pyroglutamic aciduria from vigabatrin. Lancet 1989;1:1452-3.

10 Hundemer GL, Fenves AZ. Acquired 5-oxoproline acidemia successfully treated with N-acetylcysteine. Proc (Bayl Univ Med Cent) 2017;30:169-70.

Address for correspondence: Dr William Osborne, Royal Stoke University Hospital Newcastle Rd, Stoke-on-Trent ST4 6QG, UK. Email: william.osborne@nhs.net 